\title{
Linearized Harmonic Balancing Approach for Accurate Solutions to the Dynamically Shifted Oscillator
}

\author{
A. Beléndez ${ }^{1}$, J. J. Rodes ${ }^{1}$, R. Fuentes ${ }^{2}$, C. García ${ }^{2}$, M. S. Yebra ${ }^{1}$ and I. \\ Pascual $^{2}$
}

1. Departamento de Física, Ingeniería de Sistemas y Teoría de la Señal. Universidad de Alicante. Apartado 99. E-03080 Alicante. SPAIN

2. Departamento de Óptica, Farmacología y Anatomía. Universidad de Alicante. Apartado 99. E-03080 Alicante. SPAIN

E-mail: a.belendez@ua.es, Fax:+34-96-5909750

\begin{abstract}
The analytical approximate technique developed by $\mathrm{Wu}$ et al for conservative oscillators with odd nonlinearity is used to construct approximate frequency-amplitude relations and periodic solutions to the dynamically shifted oscillator. This nonlinear oscillator is described by an equation of motion which includes a linear restoring force and an anti-symmetric, constant force which is a nonlinear force depending only upon the sign of the displacement. By combining Newton's method with the method of harmonic balance, analytical approximations to the oscillation frequency and periodic solutions are constructed for this oscillator and the approximate periods obtained are valid for the complete range of oscillation amplitudes. Excellent agreement of the approximate frequencies and periodic solutions with the exact ones are demonstrated and discussed and the results reveal that this technique is very effective and convenient for solving this class of conservative nonlinear oscillatory systems.
\end{abstract}

Keywords: Nonlinear oscillator; Analytical approximations; Harmonic balance method

\section{Introduction}

Even though the harmonic oscillator plays a major and vital role in the description of many real world processes, most of physical and mechanical oscillatory systems are often governed by nonlinear differential equations [13]. It is very difficult to solve nonlinear problems and, in general, it is often more difficult to get an analytic approximation than a numerical one for a given nonlinear problem. In particular the study of nonlinear oscillators is of great interest to many researchers.

A large variety of approximate methods is commonly used for solving nonlinear oscillatory systems. The most commons and most widely studied methods of all approximation methods for nonlinear differential equations are perturbation methods [1]. Some of other techniques include variational and variational iteration methods [4-12], exp-function [13, 14], homotopy perturbation [15-22], equivalent linearization [23, 24], standard and modified Lindstedt-Poincaré [25-29], artificial parameter $[30,31]$, parameter expanding [32-34], harmonic balance methods $[1,35-40]$, etc. Surveys of the literature with numerous references and useful bibliography and a review of these methods can be found in detail in [28] and [41].

The method of harmonic balance is a wellestablished procedure for determining analytical approximations to the solutions of differential equations, the time domain response of which can be expressed as a Fourier series. In the usual harmonic balance methods, the solution of a nonlinear system is assumed to be of the form of a truncated Fourier series [1]. Being different from the other non-linear analytical methods, such as perturbation techniques, the harmonic 
balance method does not depend on small parameters, such that it can find wide application in nonlinear problems without linearization or small perturbations and it can be also applied to intrinsically nonlinear oscillators.

To applied the harmonic balance method to the dynamically shifted oscillator [2, 42, 43], we use the analytical approach developed $\mathrm{Wu}$ et al [37] which incorporates salient features of both Newton's method and the harmonic balance method. Wu et al's approach is established by successfully linearizing the governing equation and, subsequently, appropriately imposing the harmonic balance method in order to obtain linear algebraic equations, which can be easily solved. Doing this, the complexity of the harmonic balance method is greatly simplified. We will see that the approximate solutions obtained for the dynamically shifted oscillator using this approach are valid for small as well as large amplitude of oscillation.

\section{Solution procedure}

Consider the following single degree-offreedom conservative nonlinear oscillator with displacement $x$ and mass $m$, with the Hamiltonian

$$
H(x)=\frac{1}{2} m\left(\frac{\mathrm{d} x}{\mathrm{dt}}\right)^{2}+U(x)
$$

where the potential energy is given by

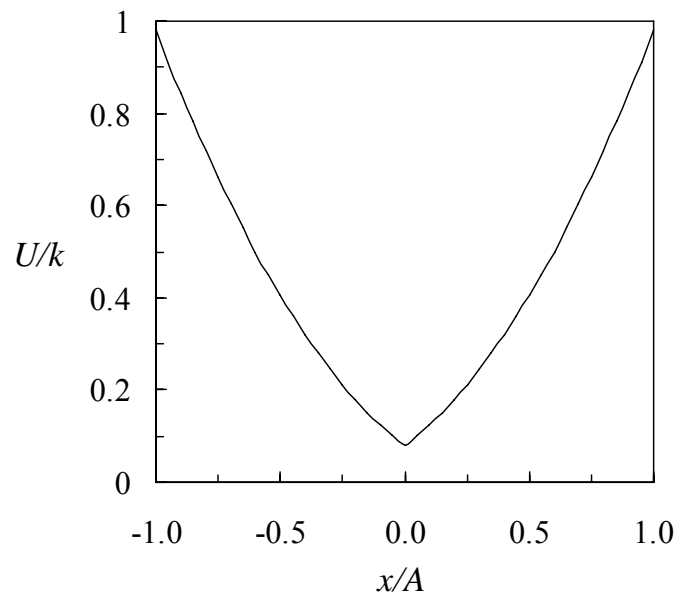

(a)

$$
U(x)=\left\{\begin{array}{l}
\frac{1}{2} k\left(x+x_{0}\right)^{2}, x \geq 0 \\
\frac{1}{2} k\left(x-x_{0}\right)^{2}, x<0
\end{array}\right.
$$

Parameter $x_{0}$ is the dynamic shift and if $x_{0}=0$ then Eq. (1) describes a simple harmonic oscillator. However, if $x_{0} \neq 0$, Eq. (1) describes a nonlinear oscillator called dynamically shifted oscillator [2]

The potential energy $U(x)$ is a continuous function of the displacement $x$, but its first derivative (the restoring force) is not. The restoring force, $F(x)=-k x-k x_{0} \operatorname{sgn}(x)$, is a nonlinear function of $x$ with a discontinuity in $x$ $=0$, and therefore the second Newton's law for the oscillator includes a discontinuity at the origin

$$
\frac{\mathrm{d}^{2} x}{\mathrm{dt}^{2}}+\omega_{0}^{2}\left[x+x_{0} \operatorname{sgn}(x)\right]=0
$$

where $\omega_{0}=\sqrt{k / m}$ and $\operatorname{sgn}(x)=x /|x|$ for $x \neq 0$ and $\operatorname{sgn}(x)=0$ for $x=0$. Parameter $s=k x_{0}$ is the stiffness parameter (finite restoring force for zero displacement) [2].

Potential energies and restoring forces relationships for the cases $x_{0}>0$ and $x_{0}<0$ are shown in Figures 1 and 2, respectively.

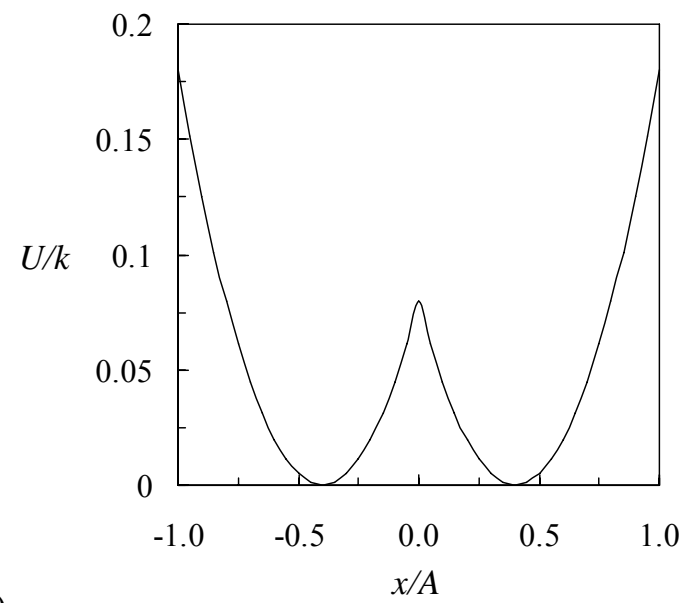

(b)

Fig. 1. Potential energies $(U / k)$ as a function of $x / A$ for the dynamically shifted oscillator with (a) $x_{0}=0.4 \mathrm{~A}$. and (b) $x_{0}=-0.4 \mathrm{~A}$. 

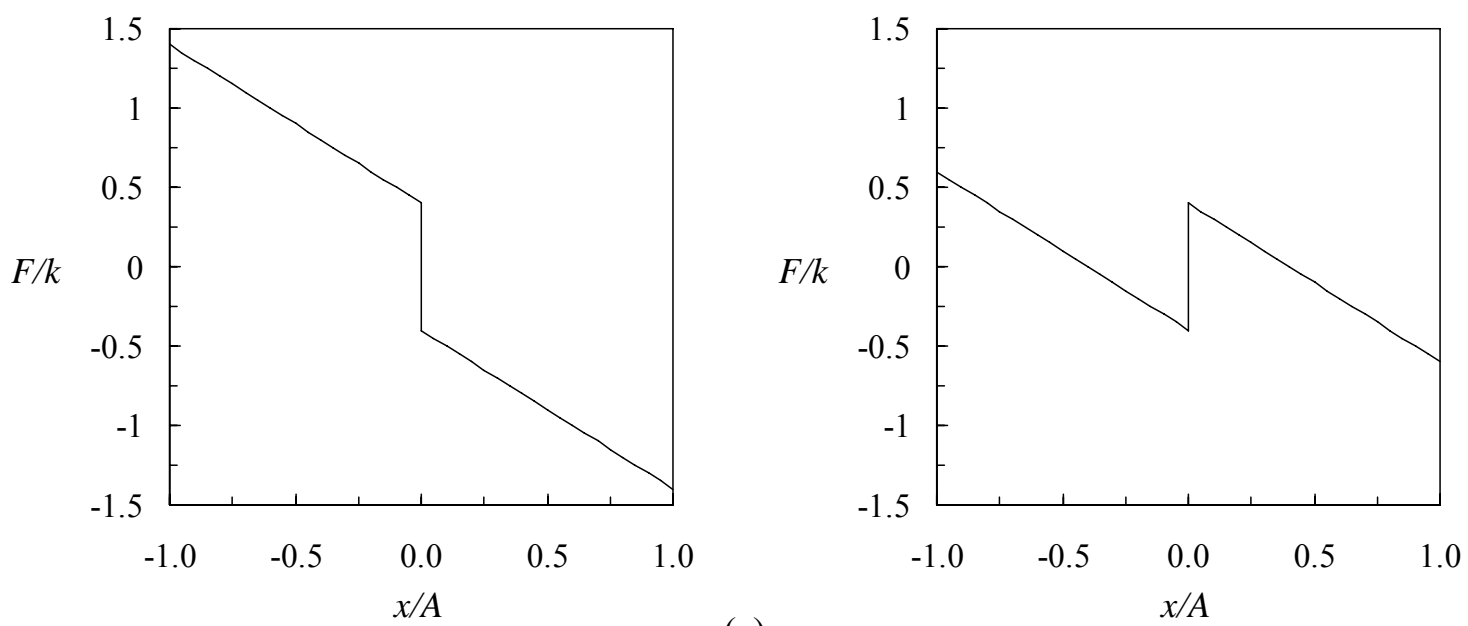

(a)

Fig. 2. Restoring force $(F / k)$ as a function of $x / A$ for the dynamically shifted oscillator with (a) $x_{0}=0.4 \mathrm{~A}$. and (b) $x_{0}=-0.4 \mathrm{~A}$.

Eq. (3) can be re-written as follows

$$
\frac{\mathrm{d}^{2} x}{\mathrm{dt}^{2}}+f(x)=0, \quad f(x)=\omega_{0}^{2}\left[x+x_{0} \operatorname{sgn}(x)\right]
$$

with initial conditions

$$
x(0)=A, \quad \frac{\mathrm{d} x(0)}{\mathrm{dt}}=0
$$

The nonlinear function $f(x)$ in Eq. (4) is odd, i.e., $f(-x)=-f(x)$, and satisfies $x f(x)>0$ for $x \in[-A, A], \quad x \neq 0 . \quad$ The equilibrium position is $x=0$, the system oscillates between symmetric bounds $[-A,+A]$, and its frequency and the corresponding periodic solution are dependent on the amplitude $A$.

For this nonlinear problem, the exact dimensionless frequency is $[2,42]$

$$
\omega_{e}(A)=\left[1-\frac{2}{\pi} \sin ^{-1}\left(\frac{x_{0}}{A+x_{0}}\right)\right]^{-1}
$$

which has a solution for all values $x_{0}>0$ and for $x_{0}<0$ only if it satisfies that $A \geq 2\left|x_{0}\right|$.

For small $x$ Eq. (3) approximates that of a nonlinear linear oscillator

$$
\frac{\mathrm{d}^{2} x}{\mathrm{dt}^{2}}+\omega_{0}^{2} x_{0} \operatorname{sgn}(x) \approx 0, \quad \text { for } \quad x<<1
$$

so, for small $A$, the frequency is $\pi \omega_{0} \sqrt{x_{0} / 8 A}$ [1], which is only valid for $x_{0}>0$. We can see that in this situation the effect of the nonlinearity is to increase the restoring force (see Fig. 2a), and hence to increase the oscillation frequency [2].

If $x_{0}<0$ both the restoring force as the oscillator frequency are decreased from their large-amplitude values [2]. For $x_{0}<0$ we have that the small value for $A$ is $2\left|x_{0}\right|$ and from Eq. (5) we obtain

$$
\lim _{A \rightarrow 2\left|x_{0}\right|} \omega_{e}(A)=\frac{1}{2}
$$

For very large $x$ (i.e. $A>>x_{0}$ ) the linear term in Eq. (3) has a large magnitude and dominates the right-hand side of the equation [2] and therefore Eq. (3) approximates that of an harmonic oscillator

$$
\frac{\mathrm{d}^{2} x}{\mathrm{dt}^{2}}+\omega_{0}^{2} x \approx 0, \quad \text { for } \quad x>>1
$$


In this limit the oscillator frequency is simply $\omega_{0}=\sqrt{k / m}$.

Then, for $x_{0}>0$ the dimensionless frequency decreases from infinity to one when the oscillation amplitude $A$ increases from zero to infinity, whereas for $x_{0}<0$ the dimensionless frequency increases from $1 / 2$ to one when the oscillation amplitude increases from $2\left|x_{0}\right|$ to infinity.

The harmonic balance method can now be applied to obtain approximations to the periodic solutions of Eq. (3). Introducing a new independent variable $\tau=\omega \omega_{0} t$, the second order differential equation can be re-written as follows

$$
\omega^{2} \frac{\mathrm{d}^{2} x}{\mathrm{~d} \tau^{2}}+x+x_{0} \operatorname{sgn}(x)=0
$$

where $\omega$ is the dimensionless frequency of the nonlinear oscillator. The new independent variable is chosen such that the solution of Eq. (9) is a periodic function of $\tau$ of period $2 \pi$. Firstly we write Eq. (9) as follows

$$
\Omega \frac{d^{2} x}{d \tau^{2}}+x+x_{0} \operatorname{sgn}(x)=0
$$

where $\Omega=\omega^{2}$. Since the restoring force is an odd function of $x$, the periodic solution $x(\tau)$ has the following Fourier series representation

$$
x(\tau)=\sum_{n=0}^{\infty} a_{2 n+1} \cos [(2 n+1) \tau]
$$

which contains only odd multiples of $\tau$.

\subsection{First-order approximation}

Following the lowest order harmonic balance method, a reasonable and simple initial approximation satisfying the conditions in Eq. (11) would be

$$
x_{1}(\tau)=A \cos \tau
$$

Substituting Eq. (12) into Eq. (10)

$$
(-\Omega+1) A \cos \tau+x_{0} \operatorname{sgn}(\cos \tau)=0
$$

It is possible to do the following Fouries series expansion

$$
\operatorname{sgn}(\cos \tau)=\sum_{n=0}^{\infty} h_{2 n+1} \cos [(2 n+1) \tau]
$$

where

$h_{2 n+1}=\frac{4}{\pi} \int_{0}^{\pi / 2} \operatorname{sgn}(\cos \tau) \cos [(2 n+1) \tau] \mathrm{d} \tau=\frac{(-1)^{n} 4}{(2 n+1) \pi}$

Substituting Eq. (14) into Eq. (13) and simplifying gives

$$
\left(-\Omega+1+\frac{4 x_{0}}{\pi A}\right) A \cos \tau+H O H=0
$$

where $\mathrm{HOH}$ stands for higher-order harmonics. Setting the coefficient of $\cos \tau$ equal to zero gives the first analytical approximate value for $\Omega_{1}$ as a function of $A$

$$
\Omega_{1}(A)=1+\frac{4 x_{0}}{\pi A}
$$

Therefore, the first analytical approximate frequency is

$$
\omega_{1}(A)=\sqrt{\Omega_{1}(A)}=\sqrt{1+\frac{4 x_{0}}{\pi A}}
$$

\subsection{Second-order approximation}

The harmonic balance method is very difficult to construct higher-order analytical approximations because it requires analytical solutions of sets of complicated nonlinear algebraic equations. To improve this method, $\mathrm{Wu}$ et al [37] presented an approach obtained by combining Newton's method with the harmonic balance method. This method is established by successfully linearizing the governing equation and, subsequently, appropriately imposing the harmonic balance method in order to obtain linear algebraic equations instead of non-linear algebraic equations.

Now $x_{1}(\tau)$ and $\Omega_{1}(A)$ are used as initial approximations to the solution of Eq. (10), and following $\mathrm{Wu}$ et al's method, the first step is the Newton procedure. The periodic solution and 
the square of frequency of Eq. (10) can be expressed as

$$
x_{2}(\tau)=x_{1}(\tau)+\Delta x_{1}(\tau), \Omega_{2}=\Omega_{1}+\Delta \Omega_{1}
$$

Substituting Eq. (19) into Eq. (10) and linearizing with respect to the correction terms $\Delta x_{1}$ and $\Delta \Omega_{1}$ lead to

$$
\left(\Omega_{1}+\Delta \Omega_{1}\right) x_{1}^{\prime \prime}+\Omega_{1} \Delta x_{1}^{\prime \prime}+x_{1}+\Delta x_{1}+x_{0} \operatorname{sgn}\left(x_{1}\right)=0
$$

and

$$
\Delta x_{1}(0)=0, \Delta x_{1}^{\prime}(0)=0
$$

where $\Delta x_{1}$ is a periodic function of $\tau$ of period $2 \pi$, and both $\Delta x_{1}$ and $\Delta \Omega_{1}$ are to be determined.

The second approximation to Eq. (19), which must satisfy the initial conditions in Eq. (21), takes de form

$$
\Delta x_{1}(\tau)=c_{1}(\cos 3 \tau-\cos \tau)
$$

where $C_{1}$ is a constant which depends on $A$ and it is to be determined.

Substituting Eqs. (12) and (22) into Eq. (20), expanding the resulting expression in a trigonometric series and setting the coefficients of the resulting items $\cos \tau$ and $\cos 3 \tau$ equal to zero, respectively, yield

$$
\begin{gathered}
A-c_{1}-A \triangle \Omega_{1}+c_{1} \Omega_{1}-A \Omega_{1}+\frac{4}{\pi} x_{0}=0 \\
c_{1}-9 c_{1} \Omega_{1}-\frac{4}{3 \pi} x_{0}=0
\end{gathered}
$$

From Eq. (23) we can obtain $C_{1}$ as follows

$$
C_{1}=\frac{4 x_{0}+\pi A-\pi A \Delta \Omega_{1}-\pi A \Omega_{1}}{\pi\left(1-\Omega_{1}\right)}
$$

Substituting Eq. (25) into Eq. (24), solving for the $\Delta \Omega_{1}$ and taking into account Eq. (17) we obtain

$$
\Delta \Omega_{1}(A)=-\frac{4 x_{0}^{2}}{27 \pi x_{0} A+6 \pi^{2} A^{2}}
$$

Furthermore, $c_{1}(A)$ can be obtained by substituting Eq. (26) into Eq. (25) and the result obtained is

$$
c_{1}(A)=-\frac{x_{0} A}{27 x_{0}+6 \pi A}
$$

The corresponding second approximate periodic solution is given by

$$
\begin{gathered}
\omega_{2}(A)=\sqrt{\Omega_{2}(A)}=\sqrt{\Omega_{1}(A)+\Delta \Omega_{1}(A)}= \\
=\sqrt{\frac{104 x_{0}^{2}+51 \pi x_{0} A+6 \pi^{2} A^{2}}{27 \pi x_{0} A+6 \pi^{2} A^{2}}}
\end{gathered}
$$

$$
x_{2}(\tau)=\left[A-c_{1}(A)\right] \cos \tau+c_{1}(A) \cos 3 \tau
$$

where $\tau=\omega_{1}(A) \omega_{0} t$.

\subsection{Third-order approximation}

To construct the third-order approximation we express the periodic solution and the square of frequency of Eq. (10) as follows

$$
x_{3}(\tau)=x_{1}(\tau)+\Delta x_{2}(\tau), \quad \Omega_{3}=\Omega_{2}+\Delta \Omega_{2}
$$

where

$$
\Delta x_{2}(\tau)=d_{1}(\cos 3 \tau-\cos \tau)+d_{2}(\cos 5 \tau-\cos \tau)
$$

where $d_{1}$ and $d_{2}$ are two constants to be determined.

Firstly we substitute Eq. (30) into Eq. (10) and we linearize the resulting equation with respect to the correction terms $\Delta \Omega_{2}$ and $\Delta x_{2}$, and secondly we substitute Eq. (31) into the resulting equation. The resulting expression is then expanding in a trigonometric series and setting the coefficients of $\cos \tau, \cos 3 \tau$ and $\cos 5 \tau$ equal to zero, respectively, yield 


$$
\begin{gathered}
\frac{4 x_{0}}{\pi}-\Delta \Omega_{2} A+\left(1-\Omega_{2}\right)\left(A-d_{1}-d_{2}\right)=0 \\
-\frac{4 x_{0}}{3 \pi}+\left(1-9 \Omega_{2}\right) d_{1}=0 \\
\frac{4 x_{0}}{5 \pi}-25 \Omega_{2} d_{2}=0
\end{gathered}
$$

which can be simultaneously solved to obtain the following expression

$\omega_{3}(A)=\sqrt{\Omega_{3}(A)}=\sqrt{\Omega_{2}+\Delta \Omega_{2}}=\sqrt{\frac{J_{1}(A)}{J_{2}(A)}}$

where

$$
\begin{aligned}
J_{1}(A)= & \alpha_{0} x_{0}^{6}+\alpha_{1} x_{0}^{5} A+\alpha_{2} x_{0}^{4} A^{2}+\alpha_{3} x_{0}^{3} A^{3}+ \\
& +\alpha_{4} x_{0}^{2} A^{4}+\alpha_{5} x_{0} A^{5}+\alpha_{6} A^{6} \\
J_{2}(A)= & \beta_{1} x_{0}^{5} A+\beta_{2} x_{0}^{4} A^{2}+\beta_{3} x_{0}^{3} A^{3}+ \\
& +\beta_{4} x_{0}^{2} A^{4}+\beta_{5} x_{0} A^{5}+\beta_{6} A^{6}
\end{aligned}
$$

$$
\begin{array}{ll}
\alpha_{0}=306828288 & \beta_{1}=78975000 \pi \\
\alpha_{1}=444396992 \pi & \beta_{2}=93543147 \pi^{2} \\
\alpha_{2}=265424547 \pi^{2} & \beta_{3}=43747938 \pi^{3} \\
\alpha_{3}=83661282 \pi^{3} & \beta_{4}=10099044 \pi^{4} \\
\alpha_{4}=14675652 \pi^{4} & \beta_{5}=1151064 \pi^{5} \\
\alpha_{5}=1358424 \pi^{5} & \beta_{6}=51840 \pi^{6} \\
\alpha_{6}=51840 \pi^{6} &
\end{array}
$$

The value for $d_{1}$ is

$$
d_{1}(A)=-\frac{H_{1}(A)}{H_{2}(A)}
$$

where

$$
\begin{aligned}
H_{1}(A)= & \gamma_{1} x_{0}^{7} A+\gamma_{2} x_{0}^{6} A^{2}+\gamma_{3} x_{0}^{5} A^{3}+\gamma_{4} x_{0}^{4} A^{4}+ \\
& +\gamma_{5} x_{0}^{3} A^{5}+\gamma_{6} x_{0}^{2} A^{6}+\gamma_{7} x_{0} A^{7}
\end{aligned}
$$

$$
\begin{aligned}
& H_{2}(A)=\chi_{0} x_{0}^{7}+\chi_{1} x_{0}^{6} A+\chi_{2} x_{0}^{5} A^{2}+\chi_{3} x_{0}^{4} A^{3}+ \\
& +\chi_{4} x_{0}^{3} A^{4}+\chi_{5} x_{0}^{2} A^{5}+\chi_{6} x_{0} A^{6}+\chi_{7} A^{7}(40)
\end{aligned}
$$

and the coefficients are given as follows

$$
\begin{array}{ll}
\gamma_{1}=4712058000 & \chi_{0}=123581250000 \\
\gamma_{2}=6776899376 \pi & \chi_{1}=206752952250 \pi \\
\gamma_{3}=4023412875 \pi^{2} & \chi_{2}=147086587830 \pi^{2} \\
\gamma_{4}=1262088936 \pi^{3} & \chi_{3}=57675372300 \pi^{3} \\
\gamma_{5}=220628124 \pi^{5} & \chi_{4}=13462160580 \pi^{4} \\
\gamma_{6}=20382192 \pi^{6} & \chi_{5}=1870477920 \pi^{5} \\
\gamma_{7}=777600 \pi^{7} & \chi_{6}=143253360 \pi^{6} \\
& \chi_{7}=4665600 \pi^{7}
\end{array}
$$

The value for $d_{2}$ is

$$
d_{2}(A)=\frac{3 x_{0} A\left(9 x_{0}+2 \pi A\right)}{10\left(325 x_{0}^{2}+156 \pi x_{0} A+18 \pi^{2} A^{2}\right)}
$$

We have taken into account Eqs. (22) and (30) to obtain Eqs. (35)-(41).

\section{Results and discussion}

We illustrate the accuracy of the modified approach by comparing the approximate solutions previously obtained with the exact solution.

For this nonlinear problem, the exact frequency is given by Eq. (5) and the periodic solution is [2]

$$
x_{e}(t)=\left(A+x_{0}\right) \cos \omega_{0} t-x_{0}, \text { for } 0 \leq t \leq \frac{T_{e}}{4}
$$

$$
\begin{array}{r}
x_{e}(t)=\left(A+x_{0}\right) \cos \left(\omega_{0} t+2 \phi\right)+x_{0}, \\
\text { for } \frac{T_{e}}{4} \leq t \leq \frac{3 T_{e}}{4}
\end{array}
$$

$$
x_{e}(t)=\left(A+x_{0}\right) \cos \left(\omega_{0} t+4 \phi\right)-x_{0},
$$

$$
\text { for } \frac{4 T_{e}}{4} \leq t \leq T_{e}
$$

where 
and

$$
T_{e}=2 \pi /\left(\omega_{0} \omega_{e}\right)
$$

$$
\phi=\sin ^{-1}\left(\frac{x_{0}}{A+x_{0}}\right)
$$

For small values of the oscillation amplitude $A$ and for $x_{0}>0$ it is possible to do the power series expansions of the exact and approximate angular frequencies. Doing these expansions, the following equations can be obtained

$$
\begin{aligned}
\omega_{e}(A) & =\frac{\pi}{2 \sqrt{2}} \sqrt{\frac{x_{0}}{A}}+\frac{5 \pi}{24 \sqrt{2}} \sqrt{\frac{A}{x_{0}}}+\ldots= \\
& =1.11072 \sqrt{\frac{x_{0}}{A}}+0.462800 \sqrt{\frac{A}{x_{0}}}+\ldots \\
\omega_{1}(A) & =\frac{2}{\sqrt{\pi}} \sqrt{\frac{x_{0}}{A}}+\frac{\sqrt{\pi}}{4} \sqrt{\frac{A}{x_{0}}}+\ldots= \\
& =1.12838 \sqrt{\frac{x_{0}}{A}}+0.443113 \sqrt{\frac{A}{x_{0}}}+\ldots \\
\omega_{2}(A) & =\frac{2}{3} \sqrt{\frac{26}{3 \pi}} \sqrt{\frac{x_{0}}{A}}+\frac{251}{108} \sqrt{\frac{\pi}{78}} \sqrt{\frac{A}{x_{0}}}+\ldots \\
& =1.10729 \sqrt{\frac{x_{0}}{A}}+0.466420 \sqrt{\frac{A}{x_{0}}}+\ldots
\end{aligned}
$$

$\omega_{3}(A)$

$$
\begin{gathered}
=\frac{8}{75} \sqrt{\frac{5122}{15 \pi}} \sqrt{\frac{x_{0}}{A}}+\frac{304122341}{4218750} \sqrt{\frac{\pi}{76830}} \sqrt{\frac{x_{0}}{A}} \\
=1.11206 \sqrt{\frac{x_{0}}{A}}+0.460971 \sqrt{\frac{A}{x_{0}}}+\ldots
\end{gathered}
$$

and therefore

$$
\begin{aligned}
& \lim _{A \rightarrow 0} \frac{\omega_{1}(A)}{\omega_{e}(A)}=\frac{4 \sqrt{2}}{\pi^{3 / 2}}=1.0159 \quad(P E=1.5 \%)(60) \\
& \lim _{A \rightarrow 0} \frac{\omega_{2}(A)}{\omega_{e}(A)}=\frac{8}{3 \pi^{3 / 2}} \sqrt{\frac{13}{3}}=0.996908
\end{aligned}
$$$$
(P E=0.31 \%)(61)
$$

$$
\lim _{A \rightarrow 0} \frac{\omega_{3}(A)}{\omega_{e}(A)}=\frac{32}{75 \pi^{3 / 2}} \sqrt{\frac{2561}{15}}=1.00121
$$

$$
(P E=0.12 \%)(62)
$$

where the percentage errors (PE) were calculated using the following equation

$$
P E(\%)=100\left|\frac{\omega_{j}-\omega_{e}}{\omega_{e}}\right| \quad j=1,2,3
$$

We can see that the relative errors for the second terms in the series expansion in Eqs. (57)-(59) are $4.3 \%, 0.78 \%$ and $0.40 \%$ for the first, the second and the third approximate frequencies, respectively. All these series expansions were carried out using MATHEMATICA.

For large values of $A$ it is possible to do the power series expansions of the exact and approximate angular frequencies, valid for all values of $x_{0}$ (for $x_{0}<0$ it is necessary that $A>2\left|x_{0}\right|$ ). Doing these expansions, the following equations can be obtained

$$
\begin{aligned}
\omega_{e}(A) & =1+\frac{2 x_{0}}{\pi A}-\frac{2(\pi-2) x_{0}^{2}}{\pi^{2} A^{2}}+\ldots \\
& =1+\frac{0.63662 x_{0}}{A}-\frac{0.231335 x_{0}^{2}}{A^{2}}+\ldots
\end{aligned}
$$

$$
\begin{aligned}
\omega_{1}(A) & =1+\frac{2 x_{0}}{\pi A}-\frac{2 x_{0}^{2}}{\pi^{2} A^{2}}+\ldots \\
& =1+\frac{0.63662 x_{0}}{A}-\frac{0.202642 x_{0}^{2}}{A^{2}}+\ldots
\end{aligned}
$$

$$
\begin{aligned}
\omega_{2}(A) & =1+\frac{2 x_{0}}{\pi A}-\frac{7 x_{0}^{2}}{3 \pi^{2} A^{2}}+\ldots= \\
& =1+\frac{0.63662 x_{0}}{A}-\frac{0.236416 x_{0}^{2}}{A^{2}}+\ldots
\end{aligned}
$$

$$
\begin{aligned}
\omega_{3}(A) & =1+\frac{2 x_{0}}{\pi A}-\frac{34 x_{0}^{2}}{15 \pi^{2} A^{2}}+\ldots= \\
& =1+\frac{0.63662 x_{0}}{A}-\frac{0.229661 x_{0}^{2}}{A^{2}}+\ldots
\end{aligned}
$$

Therefore we have 


$$
\lim _{A \rightarrow \infty} \frac{\omega_{1}(A)}{\omega_{e}(A)}=\lim _{A \rightarrow \infty} \frac{\omega_{2}(A)}{\omega_{e}(A)}=\lim _{A \rightarrow \infty} \frac{\omega_{3}(A)}{\omega_{e}(A)}=1
$$

These series expansions were carried out using MATHEMATICA. As can be seen, for large values of $A$ the first two terms in the series expansions of the approximate frequencies are the same as the first two terms obtained from the expansion of the exact frequency (Eq. (64)), whereas the third term of the expansion of the exact frequency is 0.231335 compared with $0.202642,0.236416$ and 0.229661 obtained in this study, that is, the relative errors in this term are $12 \%, 2.2 \%$ and $0.72 \%$ for the first, the second and the third approximate frequencies, respectively.

For $x_{0}<0$ we have previously seen that it is necessary that $A \geq 2\left|x_{0}\right|$ and we can obtain the following limits when $A$ tends to $2\left|x_{0}\right|$

$$
\lim _{A \rightarrow 2\left|x_{0}\right|} \omega_{e}(A)=\frac{1}{2}
$$

$$
\begin{aligned}
& \lim _{A \rightarrow 2\left|x_{0}\right|} \frac{\omega_{1}(A)}{\omega_{e}(A)}=1.20562 \quad(P E=21 \%) \\
& \lim _{A \rightarrow 2\left|x_{0}\right|} \frac{\omega_{2}(A)}{\omega_{e}(A)}=1.10250 \quad(P E=10 \%) \\
& \lim _{A \rightarrow 2\left|x_{0}\right|} \frac{\omega_{3}(A)}{\omega_{e}(A)}=1.06438 \quad(P E=6.4 \%)
\end{aligned}
$$

It can be observed that Eqs. (18), (28) and (35) provide excellent approximations to the exact frequency. It is also clear that at the third approximation order, the accuracy of the result obtained in this paper is very good and the maximum value of its percentage error for $x_{0}>0$ is less than $0.12 \%$ and tends to zero when $A$ tends to infinity.

Comparison of the exact frequency $\omega_{e}$ obtained using Eq. (5), with the proposed frequencies $\omega_{1}, \omega_{2}$ and $\omega_{3}$ computed using Eqs. (18), (28) and (55) is shown in Tables 1 and 2 for $x_{0}=4$ and $x_{0}=-4$, respectively.

Table 1 Approximate and exact frequencies for $x_{0}=4$

\begin{tabular}{lcclclcl}
\hline$A$ & $\omega_{e}$ & $\omega_{1}$ & $P E(\%)$ & $\omega_{2}$ & $P E(\%)$ & $\omega_{3}$ & $P E(\%)$ \\
\hline 0.1 & 7.09758 & 7.20622 & 1.5 & 7.07642 & 0.30 & 7.10576 & 0.12 \\
0.4 & 3.65557 & 3.70572 & 1.4 & 3.64576 & 0.27 & 3.65928 & 0.10 \\
0.7 & 2.84159 & 2.87674 & 1.2 & 2.83470 & 0.24 & 2.84415 & 0.090 \\
1 & 2.44102 & 2.46839 & 1.1 & 2.43564 & 0.22 & 2.44299 & 0.081 \\
4 & 1.50000 & 1.50773 & 0.52 & 1.49849 & 0.10 & 1.50051 & 0.034 \\
7 & 1.31050 & 1.31437 & 0.30 & 1.30975 & 0.057 & 1.31074 & 0.019 \\
10 & 1.22618 & 1.22853 & 0.19 & 1.22573 & 0.037 & 1.22633 & 0.012 \\
100 & 1.02511 & 1.02515 & 0.0041 & 1.02510 & 0.00074 & 1.02511 & 0.00024 \\
\hline
\end{tabular}

Table 2 Approximate and exact frequencies for $x_{0}=-4$

\begin{tabular}{lcclclcl}
\hline$A$ & $\omega_{e}$ & $\omega_{1}$ & $P E(\%)$ & $\omega_{2}$ & $P E(\%)$ & $\omega_{3}$ & $P E(\%)$ \\
\hline 8 & 0.500000 & 0.602810 & 21 & 0.551251 & 10 & 0.532190 & 6.4 \\
10 & 0.682799 & 0.700503 & 2.6 & 0.682200 & 0.088 & 0.683795 & 0.15 \\
16 & 0.822134 & 0.825645 & 0.43 & 0.821653 & 0.059 & 0.822327 & 0.024 \\
20 & 0.861429 & 0.863338 & 0.22 & 0.861142 & 0.033 & 0.861536 & 0.012 \\
30 & 0.910471 & 0.911172 & 0.077 & 0.910357 & 0.013 & 0.910511 & 0.0044 \\
40 & 0.933810 & 0.934171 & 0.039 & 0.933749 & 0.0065 & 0.933830 & 0.0022 \\
50 & 0.947483 & 0.947703 & 0.023 & 0.947445 & 0.0040 & 0.947495 & 0.0013 \\
100 & 0.974152 & 0.974202 & 0.0052 & 0.974144 & 0.00090 & 0.974155 & 0.00030 \\
\hline
\end{tabular}


The normalized exact periodic solution $x_{e} / A$ in Eq. (55) and the third-order approximate periodic solution, $x_{3} / A$, is plotted in Figures 3-5 for different values of $x_{0}$, whereas in Figures 6-8 we plotted the difference $\Delta=\left(x_{e}-x_{3}\right) / A$. In these figures parameter $h$

$$
h=\frac{\omega_{0} \omega_{e}(A) t}{2 \pi}
$$

is defined as

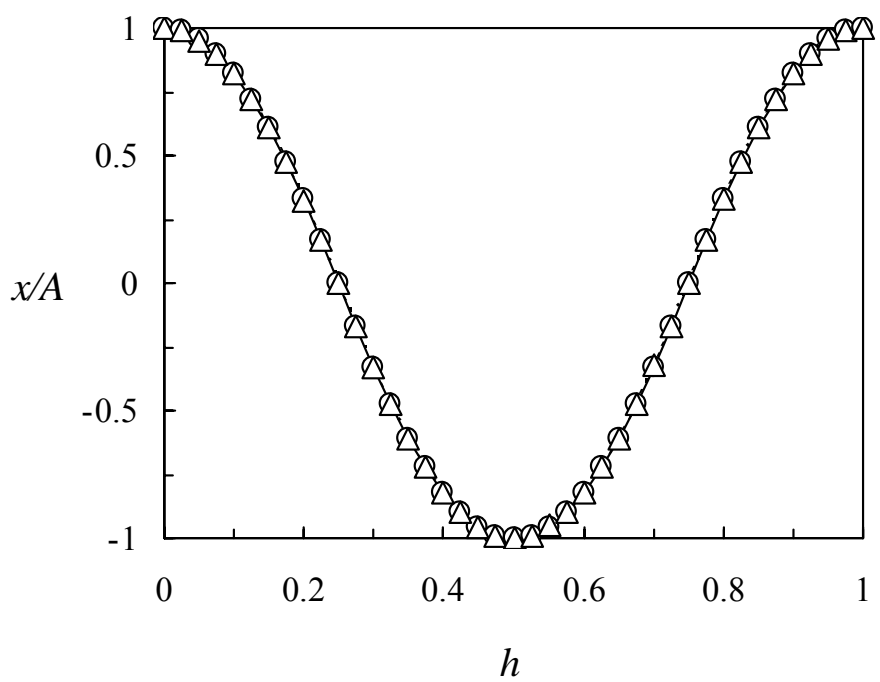

Fig. 3. Comparison of the approximate third-order solution (dashed line and triangles) with the exact solution (continuous line and circles) for $x_{0}=0.4 \mathrm{~A}$.

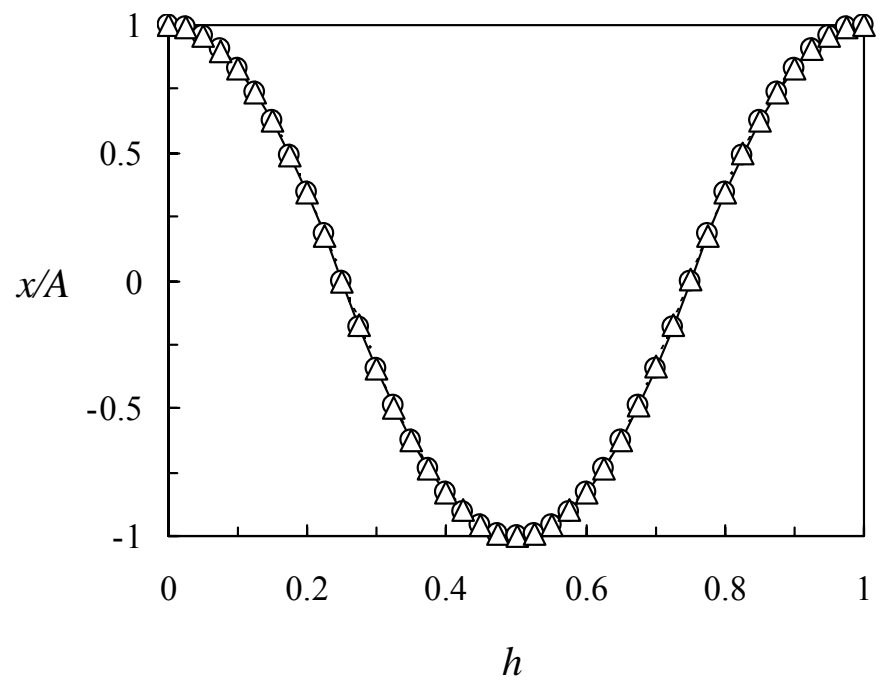

Fig. 4. Comparison of the approximate third-order solution (dashed line and triangles) with the exact solution (continuous line and circles) for $x_{0}=1.6 \mathrm{~A}$. 


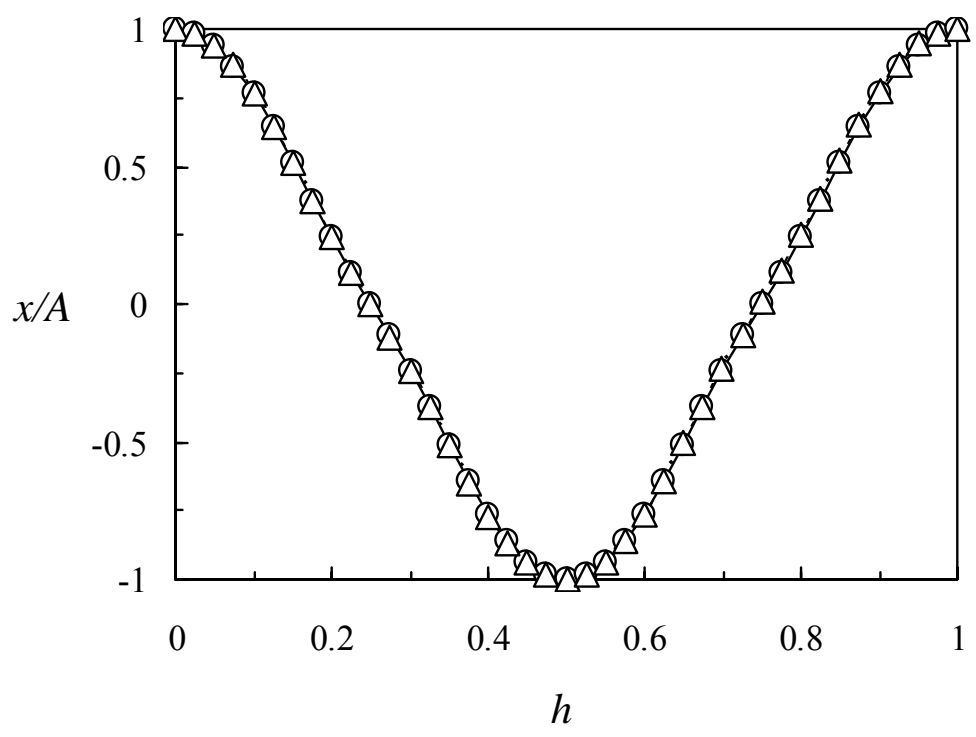

Fig. 5. Comparison of the approximate third-order solution (dashed line and triangles) with the exact solution (continuous line and circles) for $x_{0}=-0.4 \mathrm{~A}$.

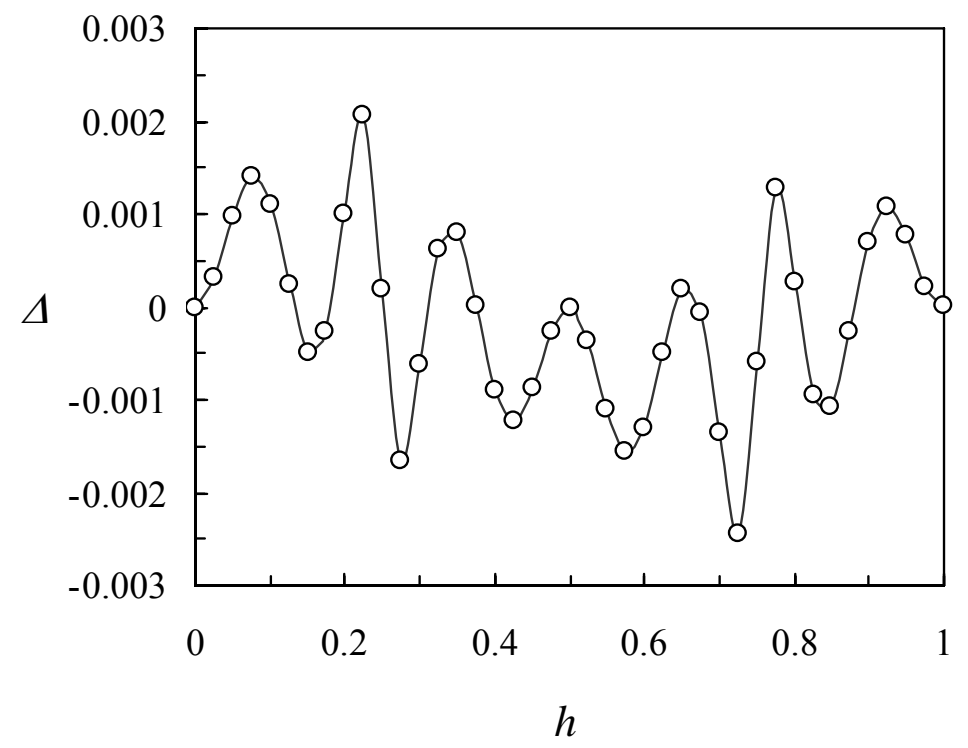

Fig. 6. Difference between normalized exact and approximate third-order solutions for $x_{0}=0.4 \mathrm{~A}$. 


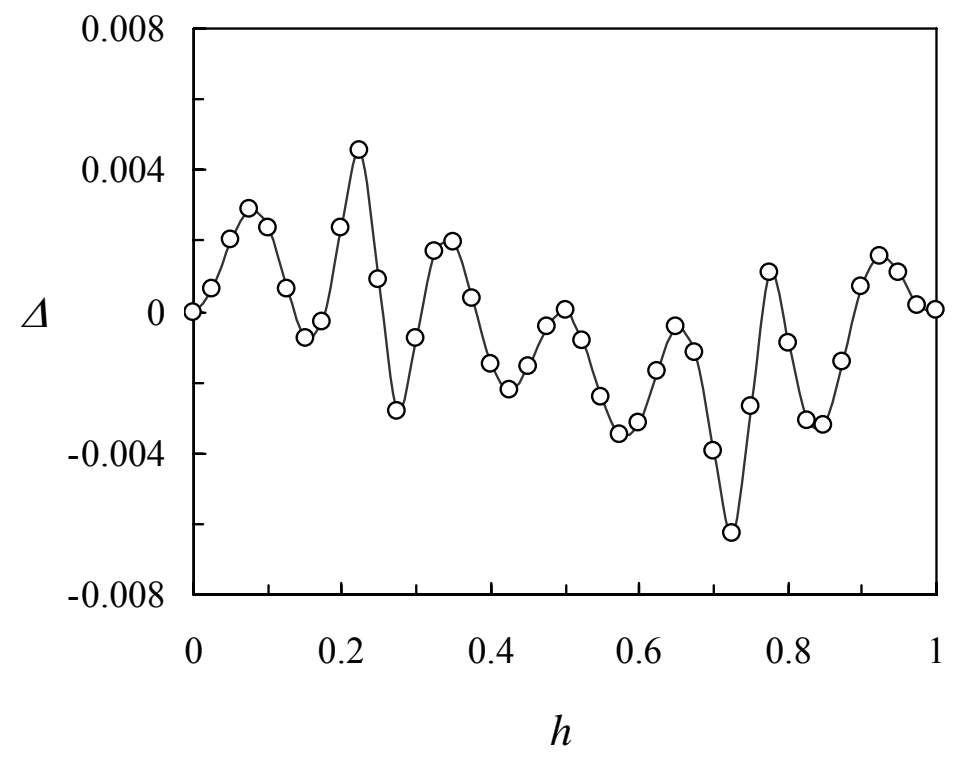

Fig. 7. Difference between normalized exact and approximate third-order solutions for $x_{0}=1.6 \mathrm{~A}$.

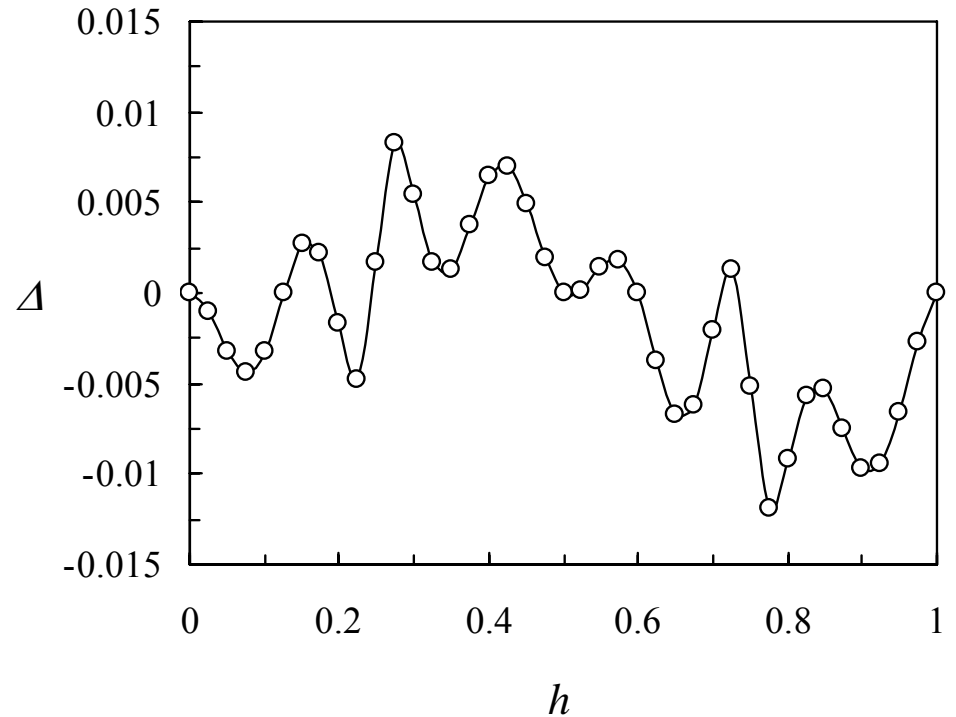

Fig. 8. Difference between normalized exact and approximate third-order solutions for $x_{0}=-0.4 \mathrm{~A}$.

An easy and direct calculation gives the following series representation for the exact solution $x_{e}(t)$ (Eq. (54))

$$
x_{e}(t)=\sum_{n=0}^{\infty} a_{2 n+1} \cos \left[(2 n+1) \omega_{e} t\right]
$$

The first terms of the Fourier expansion in Eq. (74) are

$$
a_{1}=\frac{x_{0}}{4(\pi-2 S)}\left[\frac{96 C^{3}}{\pi^{3}-4 \pi C^{2}}+\frac{(\pi-2 S)^{3}}{\pi(\pi-S) S}\right]
$$


$a_{2}=-\frac{x_{0}}{12(\pi-2 S)}\left[\frac{96 C^{3}}{9 \pi^{3}-4 \pi C^{2}}+\frac{(\pi-2 S)^{3}}{\pi\left(2 \pi^{2}+\pi S-S^{2}\right)}\right]$

$a_{3}=\frac{x_{0}}{20(\pi-2 S)}\left[\frac{96 C^{3}}{25 \pi^{3}-4 \pi C^{2}}+\frac{(\pi-2 S)^{3}}{\pi\left(6 \pi^{2}+\pi S-S^{2}\right)}\right]$

where $C$ and $S$ are given as follows

$$
\begin{gathered}
C=\cos ^{-1}\left(\frac{x_{0}}{A+x_{0}}\right) \\
S=\sin ^{-1}\left(\frac{x_{0}}{A+x_{0}}\right)
\end{gathered}
$$

Taking into account Eqs. (12), (30) and (31) it is possible to write the third order approximate solution as follows

$$
\left.x_{3}(t)=\sum_{n=0}^{3} b_{2 n+1} \cos \left[(2 n+1) \omega_{0} \omega_{3} t\right)\right]
$$

where

$$
\begin{gathered}
b_{1}=A-d_{1}-d_{2} \\
b_{3}=d_{1} \\
b_{5}=d_{2}
\end{gathered}
$$

In Tables 3-8 we compare $b_{1}, b_{3}$ and $b_{5}$ with the first three terms of the Fourier expansion of $x_{e}(t)$ (Eqs. (75)-(77)) for different values of $x_{0}$. From these tables we can see that the relative errors for the different coefficients are small.

Table 3. Coefficients of the third order approximate solution and first three coefficients of the Fourier expansion of the exact solution for $x_{0} / A=0.2$.

\begin{tabular}{l|l|l|l}
\hline$n$ & $a_{2 n+1} / A$ & $b_{2 n+1} / A$ & error \\
\hline 0 & 1.00700 & 1.00658 & $0.042 \%$ \\
1 & -0.00826036 & -0.00826211 & $0.021 \%$ \\
2 & 0.00167963 & 0.00168009 & $0.027 \%$ \\
\hline
\end{tabular}

Table 4. Coefficients of the third order approximate solution and first three coefficients of the Fourier expansion of the exact solution for $x_{0} / A=0.4$.

\begin{tabular}{l|l|l|l}
\hline$n$ & $a_{2 n+1} / A$ & $b_{2 n+1} / A$ & error \\
\hline 0 & 1.01146 & 1.01076 & $0.069 \%$ \\
1 & -0.0135468 & -0.0135490 & $0.016 \%$ \\
2 & 0.00278394 & 0.00278603 & $0.075 \%$ \\
\hline
\end{tabular}

Table 5. Coefficients of the third order approximate solution and first three coefficients of the Fourier expansion of the exact solution for $x_{0} / A=0.8$.

\begin{tabular}{l|l|l|l}
\hline$n$ & $a_{2 n+1} / A$ & $b_{2 n+1} / A$ & error \\
\hline 0 & 1.01684 & 1.01579 & $0.10 \%$ \\
1 & -0.0199542 & -0.0199517 & $0.13 \%$ \\
2 & 0.00415386 & 0.00416086 & $0.17 \%$ \\
\hline
\end{tabular}

Table 6. Coefficients of the third order approximate solution and first three coefficients of the Fourier expansion of the exact solution for $x_{0} / A=1.6$.

\begin{tabular}{l|l|l|l}
\hline$n$ & $a_{2 n+1} / A$ & $b_{2 n+1} / A$ & error \\
\hline 0 & 1.02204 & 1.02063 & $0.14 \%$ \\
1 & -0.026179 & -0.0261617 & $0.066 \%$ \\
2 & 0.0055185 & 0.0055346 & $0.29 \%$ \\
\hline
\end{tabular}

Table 7. Coefficients of the third order approximate solution and first three coefficients of the Fourier expansion of the exact solution for $x_{0} / A=-0.2$.

\begin{tabular}{l|l|l|l}
\hline$n$ & $a_{2 n+1} / A$ & $b_{2 n+1} / A$ & error \\
\hline 0 & 0.987239 & 0.987972 & $0.074 \%$ \\
1 & 0.014948 & 0.0149318 & $0.11 \%$ \\
2 & -0.00290172 & -0.00290377 & $0.071 \%$ \\
\hline
\end{tabular}

Table 8. Coefficients of the third order approximate solution and first three coefficients of the Fourier expansion of the exact solution for

\begin{tabular}{l|l|l|l}
\multicolumn{1}{l}{$x_{0} / A=-0.4}$. \\
\hline$n$ & $a_{2 n+1} / A$ & $b_{2 n+1} / A$ & error \\
\hline 0 & 0.954122 & 0.956737 & $0.27 \%$ \\
1 & 0.0531191 & 0.0528404 & $0.52 \%$ \\
2 & -0.00955942 & -0.00957782 & $0.19 \%$ \\
\hline
\end{tabular}




\section{Conclusions}

A linearized harmonic balance method proposed by $\mathrm{Wu}$ et al [37] was used to obtain two approximate frequencies for the dynamically shifted oscillator. We can conclude that the approximate frequencies obtained are valid for the complete range of oscillation amplitude, including the limiting cases of amplitude approaching zero and infinity. Excellent agreement of the approximate frequencies with the exact one was demonstrated and discussed. Some examples have been presented to illustrate excellent accuracy of the approximate analytical solutions. Finally, we can see that this method is very simple in its principle, and is very easy to be applied and it provides very accurate results for the dynamically shifted oscillator.

\section{References}

[1] R. E. Mickens, Oscillations in Planar Dynamics Systems (World Scientific, Singapore 1996).

[2] W. M. Hartmann, The dynamically shifted oscillators, Am. J. Phys. 54 (1986) 28-32.

[3] J. H. He, Non-perturbative methods for strongly nonlinear problems (dissertation.deVerlag im Internet GmbH, Berlin 2006).

[4] J. H. He, Variational approach for nonlinear oscillators, Chaos Soliton. Fract.34 (2007) 1430-1439.

[5] M. Dehghan and M. Tatari, Te use of He's variational iteration method for solving multipoint boundary value problems, Phys. Scr. 72 (2007) 672-676.

[6] J. H. He, Variational iteration method - a kind of non-linear analytical technique: some examples, Int. J. Non-linear Mech. 34 (1999) 699-708.

[7] J. H. He, X. H. Wu, Construction of solitary solution and compact on-like solution by variational iteration method, Chao, Soliton. Fract. 29 (2006) 108-113.

[8] L. Xu, Variational approach to the nonlinear oscillator of a mass attached to a stretched elastic wire, Phys. Scr. 77, 045006 (2008).

[9] E. Yusufoglu, Variational iteration method for construction of some compact and noncompact structures of Klein-Gordon equations, Int. J. Nonlin. Sci. Num. 8 (2007) 153-158.
[10] A. Sadighi and D.D. Ganji, Solution of the generalized nonlinear Boussinesq equation using homotopy perturbation and variational iteration methods, Int. J. Nonlin. Sci. Num. 8 (2007) 435-443.

[11] M. A. Noor and S. T. Mohyud-Din, Variational iteration method for solving higher-order nonlinear boundary value problems using He's polynomials, Int. J. Nonlin. Sci. Num. 9 (2008) 141-156.

[12] A. Ramiar, D. D. Ganji and Q. Esmaili, Homotopy perturbation method and variational iteration method for orthogonal 2-D and axisymmetric impinging jet problems, Int. J. Nonlin. Sci. Num. 9 (2008) 115-130.

[13] T. Ozis and C. Koroglu, A novel approach for solving the Fisher equation using Exp-function method, Phys. Lett. A 372 (2008) 3836-3840.

[14] S. D. Zhu, Exp-function method for the discrete mKdV lattice, Int. J. Nonlin. Sci. Num. 8 (2007) 465-468.

[15] J. H. He, Homotopy perturbation method for solving boundary value problems, Phys. Lett. A 350 (2006) 87-88.

[16] T. Özis and A. Yildirim, A comparative study of He's homotopy perturbation method for determining frequency-amplitude relation of a nonlinear oscillator with discontinuities, Int. J. Nonlin. Sci. Num. 8 (2007) 243-248.

[17] A. Beléndez, A. Hernández, T. Beléndez, E. Fernández, M. L. Álvarez and C. Neipp, Application of He's homotopy perturbation method to the Duffing-harmonic oscillator, Int. J. Nonlin. Sci. Num.8 (2007) 79-88.

[18] A. Beléndez, A. Hernández, T. Beléndez, C. Neipp and A. Márquez, Application of the homotopy perturbation method to the nonlinear pendulum, Eur. J. Phys. 28 (2007) 93-104.

[19] F. Shakeri and M. Dehghan, Inverse problem of diffusion by He's homotopy perturbation method, Phys. Scr. 75 (2007) 551-556.

[20] M. M. Mousa and S. F. Ragab, Application of the homotopy perturbation method to linear and nonlinear Schrödinger equation, $Z$. Naturforsch. 63a (2008) 140-144.

[21] M. T. Darvishi and F. Khani, Application of He's homotopy perturbation method to stiff systems of ordinary differential equations, $Z$. Naturforsch. 63a (2008) 19-23.

[22] A. Yildirim, "Exact solutions of nonlinear differential-difference equations by He's homotopy perturbation method", Int. J. Non- 
linear Sci. Numer. Simulation 9 (2008) 111114.

[23] A. Beléndez, A. Hernández, T. Beléndez, A. Márquez and C. Neipp, An improved 'heuristic' approximation for the period of a nonlinear pendulum: linear analysis of a nonlinear problem, Int. J. Nonlin. Sci. Num. 8 (2007) 329-334.

[24] A. Beléndez, C. Pascual, C. Neipp, T. Beléndez and A. Hernández, An equivalent linearization method for conservative nonlinear oscillators, Int. J. Nonlin. Sci. Num. 9 (2008) 9-17.

[25] J. H. He, A new perturbation technique which is also valid for large parameters, J. Sound Vib. 229 (2000) 1257-1263.

[26] J. H. He, Modified Lindstedt-Poincare methods for some non-linear oscillations. Part I: expansion of a constant, Int. J. Non-linear Mech. 37 (2002) 309-314.

[27] H. M. Liu, Approximate period of nonlinear oscillators with discontinuities by modified Lindstedt-Poincaré method, Chaos Soliton. Fract. 23 (2005) 577-579.

[28] J. H. He, Some asymptotic methods for strongly nonlinear equations, Int. J. Mod. Phys. B 20 (2006) 1141-1199.

[29] J. I. Ramos, On Lindstedt-Poincaré techniques for the quintic Duffing equation, Appl. Math. Comput. 193 (2007) 303-310.

[30] J. I. Ramos, An artificial parameterdecomposition method for nonlinear oscillators: Applications to oscillators with odd nonlinearities, J. Sound Vib. 307 (2007), 312329.

[31] J. H. He, A modified perturbation technique depending upon an artificial parameter, Meccanica 35 (2000) 299-311.

[32] J. H. He, Application of parameter-expanding method to strongly nonlinear oscillators, Int. J. Nonlin. Sci. Num. 8 (2007) 121-124.

[33] S. Q. Wang and J. H. He, Nonlinear oscillator with discontinuity by parameter-expansion method, Chaos, Soliton. Fract. 35 (2008) 688691.

[34] D. H. Shou, J. H. He, Application of parameter-expanding method to strongly nonlinear oscillators, Int. J. Nonlin. Sci. Num. 8 (2007) 121-124.

[35] C.W. Lim, B.S. Wu, W.P. Sun, Higher accuracy analytical approximations to the Duffing-harmonic oscillator, J. Sound Vib. 296 (2006) 1039-1045.
[36] A. Beléndez, A. Hernández, A. Márquez, T. Beléndez and C. Neipp, Analytical approximations for the period of a simple pendulum, Eur. J. Phys. 27 (2006) 539-551.

[37] B. S. Wu, W. P. Sun and C. W. Lim, An analytical approximate technique for a class of strongly non-linear oscillators, Int. J. Nonlinear Mech. 41, 766-774 (2006).

[38] B.S. Wu, P.S. Li, A method for obtaining approximate analytic periods for a class of nonlinear oscillators, Meccanica 36 (2001) 167-176.

[39] A. Beléndez, A. Hernández, T. Beléndez, M. L. Álvarez, S. Gallego, M. Ortuño and C. Neipp, Application of the harmonic balance method to a nonlinear oscillator typified by a mass attached to a stretched wire, J. Sound Vib. 302 (2007) 1018-1029.

[40] A. Beléndez, E. Gimeno, E. Fernández, D. I. Méndez and M. L. Álvarez, Accurate approximate solution to nonlinear oscillators in which the restoring force is inversely proportional to the dependent variable, Phys. Scr. 77, 065004 (2008).

[41] J. H. He, An elementary introduction to recently developed asymptotic methods and nanomechanics in textile engineering, Int. J. Mod. Phys. B 22 (2008) 3487-3578

[42] J. S. Thomsen, A benevolent nonlinear system: The dynamically shifted oscillator, Am. J. Phys. 56 (1988) 123-128.

[43] A. Beléndez, M. L. Álvarez, E. Fernández and I. Pascual, Linearization of nonlinear oscillators, Eur. J. Phys. 30(2009) 259-270. 